\section{Drs. Rasmussen and Scofield reply}

\section{To the Editor:}

Cafaro and colleagues ${ }^{1}$ have studied focus score (FS)-negative (that is, $<1$ focus of $\geq 50$ lymphocytes per $4 \mathrm{~mm}^{2}$ ) to FS-positive ( $\geq 1$ focus per $4 \mathrm{~mm}^{2}$ ) Sjögren syndrome (SS) patients ${ }^{2}$ in a manner similar to our recent study ${ }^{3}$. As in our study, there was evidence of B cell hyperreactivity among those subjects with a positive minor salivary gland biopsy, although the details of this finding varied between the 2 studies. We found statistically significant elevations of anti-La/SSB and hypergammaglobulinemia, whereas Cafaro, et al found numerically increased anti-La/SSB and a statistically increased rheumatoid factor; the latter did not withstand correction for multiple comparisons, however.

We agree that highly sensitive autoantibody detection methods may find low-level, low-titer antibodies, the clinical significance of which is unknown ${ }^{4}$. However, we point out that we only classified our subjects and did not diagnose them. Research classification relies on a set of criteria, whereas diagnosis remains a clinical enterprise for which the gold standard is expert opinion 5 . In addition, our study was at the initial evaluation of the patients, while the studies of Cafaro, et $a l^{1}$, and Carubbi and Alunno ${ }^{6}$ were retrospective. This difference in study design may produce variation in findings.

Also, Cafaro, et al $l^{1}$ found a higher incidence of leukopenia, which is associated with the presence of anti-Ro/SSA ${ }^{4}$, among the FS $\geq 1$ subjects compared to those with $\mathrm{FS}<1$. Of course, as in our study, all these subjects had anti-Ro/SSA ${ }^{6}$. Cafaro and colleagues ${ }^{1}$ make the interesting point that their finding of leukopenia fits well with our finding of an increased incidence of an interferon (IFN) signature in the peripheral blood cells of participants with FS $\geq 1$ compared to the FS $=0$ subjects; this is because leukopenia has been associated with IFN activity and higher FS. We did not find this same association with low white blood cell counts. Perhaps, differences in study design (cross-sectional at diagnosis vs longitudinal), method of autoantibody detection, diagnosis versus classification, and/or cohort size led to this difference.

The pathogenesis of SS is poorly understood. As we stated in our study ${ }^{3}$ and is again demonstrated in the data of both Cafaro, $e t$ al $l^{1}$ and Carubbi and Alunno ${ }^{6}$, the study of FS-negative subjects may allow dissection of the pathogenic associations of $\mathrm{T}$ cell pathology (glandular infiltrate), B cell pathology (circulating autoantibodies), and innate immune pathology (IFN activity), as well as clinical manifestations of the disease.
Astrid Rasmussen ${ }^{1,2} \mathbb{D}, \mathrm{MD}, \mathrm{PhD}$

R. Hal Scofield ${ }^{3,4,5}$ (1), MD

${ }^{1}$ Genes and Human Disease Research Program, Oklahoma Medical

Research Foundation, Oklahoma City, Oklahoma, USA;

${ }^{2}$ Dirección de Investigación, Instituto Nacional de Ciencias Medicas y

Nutrición Salvador Zubirán, Mexico City, Mexico;

${ }^{3}$ Arthritis \& Clinical Immunology Program, Oklahoma Medical Research Foundation;

${ }^{4}$ Department of Medicine, College of Medicine, University of Oklahoma Health Sciences Center;

${ }^{5}$ Oklahoma City Department of Veterans Affairs Medical Center, Oklahoma City, Oklahoma, USA.

This work was supported in part by Veterans Affairs Merit Review 5I01BX001451, as well as National Institutes of Health grants AR053734, AR053483, AI082714, and GM104938.

Address correspondence to Dr. R.H. Scofield, MD, 732 NE 18th Street, Oklahoma City, OK 73104, USA. Email: hal-scofield@omrf.ouhsc.edu.

\section{REFERENCES}

1. Cafaro G, Gerli R, Bartoloni E. Association between glandular infiltrate and leukopenia in Sjögren Syndrome (SS): data from the Italian Group for Study of SS (GRISS). J Rheumatol 2020; 47:1840-41

2. Fisher BA, Jonsson R, Daniels T, Bombardieri M, Brown RM, Morgan P, et al; Sjögren's histopathology workshop group from ESSENTIAL (EULAR Sjögren's syndrome study group). Standardisation of labial salivary gland histopathology in clinical trials in primary Sjogren's syndrome. Ann Rheum Dis 2017;76:1161-8.

3. Sharma R, Chaudhari KS, Kurien BT, Grundahl K, Radfar L, Lewis DM, et al. Sjogren syndrome without focal lymphocytic infiltration of the salivary glands. J Rheumatol 2020;47:394-9.

4. Fayyaz A, Kurien BT, Scofield RH. Autoantibodies in Sjogren's syndrome. Rheum Dis Clin North Am 2016;42:419-34.

5. Shiboski CH, Shiboski SC, Seror R, Criswell LA, Labetoulle M, Lietman TM, et al; International Sjögren's Syndrome Criteria Working Group. 2016 American College of Rheumatology/ European League Against Rheumatism classification criteria for primary Sjogren's syndrome: a consensus and data-driven methodology involving three international patient cohorts. Arthritis Rheumatol 2017;69:35-45.

6. Carubbi F, Alunno A. The serological status affects the prognostic role of salivary gland histology in primary Sjögren's syndrome. J Rheumatol 2020;47:1838-9. 